\title{
"Siegesplätze über die Natur". Musils Kritik am Geist des modernen Wettkampfsports
}

\author{
ANNE FLEIG
}

Dem Sportenthusiasmus der Zwischenkriegszeit hat Robert Musil, der sein Leben lang verschiedene Sportarten ausgeübt hat, in seinem Essay Als Papa Tennis lernte ein ambivalentes Zeugnis ausgestellt. ${ }^{1}$ Während er selbst in verschiedenen Texten - seinen Essays ebenso wie in seinem unvollendeten Roman Der Mann ohne Eigenschaften - ein starkes, ästhetisches Interesse an der Sportausübung formuliert hat, blieb ihm die auf den Sport projizierte, programmatische Rhetorik von „Kraft und Schönheit “2 neuer, körpergestählter Menschen, die die vorwiegend bürgerlichen Anhänger der Körperkulturbewegung mit den Funktionären der Arbeitersportvereine verband, ständiger Anstoß zur Kritik.

1 Der Essay erschien 1931 im April-Heft der Zeitschrift Der Querschnitt (1921-1936). Das „Magazin der aktuellen Ewigkeitswerte“ - so der Untertitel der Zeitschrift - wurde in Zusammenarbeit mit der Galerie Flechtheim von Hermann von Wedderkop in Berlin herausgegeben und hatte sich unter anderem der Reflexion des Sports, insbesondere des Boxens, verschrieben. Der Querschnitt war eines der wichtigsten Organe der intellektuellen Sportbegeisterung der Weimarer Republik. Vgl. Robert Musil: Als Papa Tennis lernte, in: Der Querschnitt XI. Jhrg., Heft 4, Berlin 1931, S. 247-252. Zur Sportbetrachtung in Der Querschnitt vgl. den Beitrag von Kai Marcel Sicks in diesem Band.

2 So der Titel einer frühen Zeitschrift zur Körperkultur (1901-1927; zuerst mit dem Untertitel: Zeitschrift für vernünftige Leibeszucht, dann: Monatsschrift für moderne Körperkultur) und des populären UFA-Films „Wege zu Kraft und Schönheit“" (Regie: Wilhelm Prager, Deutschland 1924/25). 


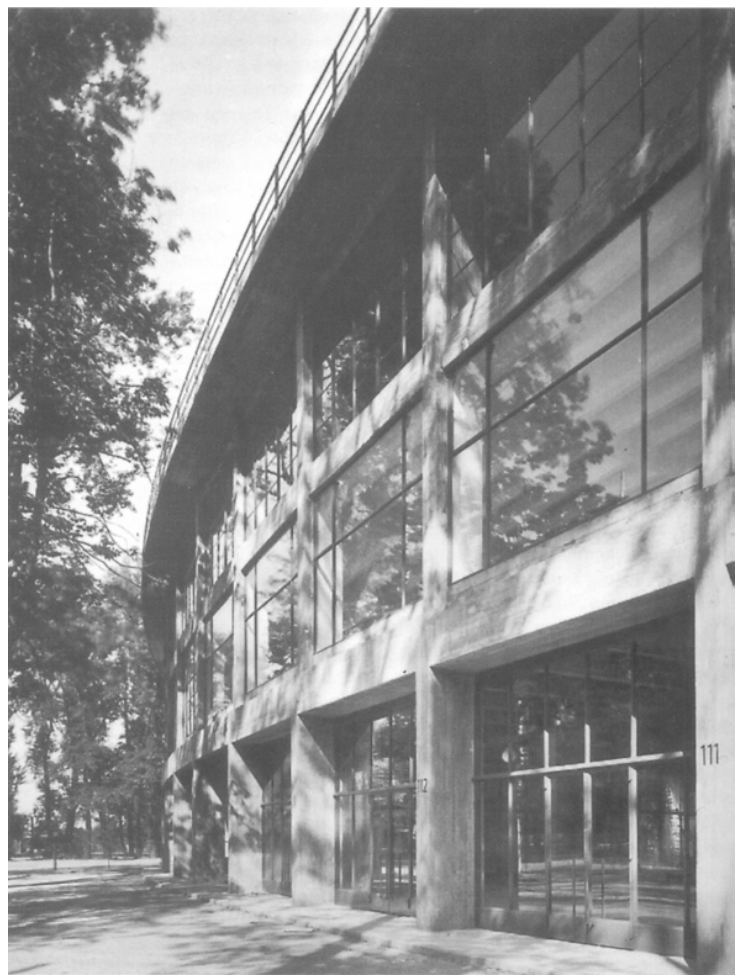

Abb. 1: Das Wiener Praterstadion im Jahr seiner Eröffnung 1931.

Ausgangspunkt seines Essays Als Papa Tennis lernte ist der von der Wiener Sozialdemokratie initiierte Bau eines olympischen Stadions im Prater, das vor allem der kollektiven Fußballleidenschaft und dem Arbeitersport im Wien der zwanziger und dreißiger Jahre Raum geben sollte und sich damit als wichtiger Beitrag zur sozialen Modernisierung und Zivilisierung der Massen verstand (Abb. 1; zur Geschichte der Pläne für dieses Stadion vom Ersten Weltkrieg bis zum Anstoß zu ihrer Umsetzung durch die Vergabe der Arbeiterolympiade an Österreich 1927 vgl. Horak/Maderthaner 1997: 34-37).

Musils Text vollzieht aus der Perspektive der Erinnerung die Herausbildung des modernen Wettkampfsportes von einem geselligen Vergnügen der oberen Schichten Ende des 19. Jahrhunderts, wie es sich etwa im Tennis, aber auch im Reiten oder Rudern manifestierte, hin zu einem öffentlichkeitswirksamen Massenspektakel in der Weimarer Republik nach und macht die damit einhergehenden Veränderungen in der 
Wahrnehmung und Zurschaustellung des Körpers im Sport anschaulich. Seine zentrale These lautet, dass erst das Zuschauen, also die institutionalisierte Inszenierung des Körperspektakels für das Publikum im Stadion, die Sportausübung zum sportlichen Großereignis gemacht habe. Gegenüber dem Sport als Teil der Massenkultur, der bereits durch bloße Zahlengröße beeindruckt - in Gestalt neuer Sportrekorde, der Länge der Sportberichterstattung und rekordverdächtiger Zuschauerzahlen -, betont Musil die Möglichkeiten des subjektiven Erlebens in der Körperbewegung und die Sinnlosigkeit des sportlichen Tuns als ästhetischen Gewinn. Dass auch Zuschauen Teilhabe an zweckfreiem Vergnügen bedeuten kann, diskutiert der Autor in Als Papa Tennis lernte nicht, da dem massenhaften Zuschauen beim Sport die Bedeutungszuschreibung seiner Ansicht nach immer schon inhärent ist.

Daher fokussiert Musils Essay nicht den Körper, sondern den Geist, der den modernen Sport hervorgebracht hat (vgl. auch Fleig 2004). Er kritisiert die diskursive Produktion von Bedeutung der eigentlich zweckfrei-spielerischen, körperlichen Bewegung. Vor dem Hintergrund der lauten Erneuerungsrhetorik seiner Gegenwart, dem kollektiven Traum von einer neuen Zeit und einem befreiten, neuen Menschen richtet bereits der bescheiden klingende Titel des Essays die Aufmerksamkeit auf die individuelle Perspektive eines Sportlers, nämlich den Tennis spielenden Vater, der als Privatperson spricht, und nicht auf einen in der Öffentlichkeit stehenden Tennisprofi. Macht der Essay damit einerseits die Sportausübung zum selbstverständlichen Teil der Familiengeschichte, was die vertrauliche Anrede „Papa“ noch unterstreicht, muss diese familiäre Haltung aus der Perspektive eines Zeitgeistmagazins von 1931 andererseits als hoffnungslos veraltete Sicht erscheinen, in der sich ironische Distanz zum Inhalt des Textes offenbart. Musil nutzt die Form des Essays, um den Geist möglicher Subjektivität in seiner Ambivalenz von Faszination und Kritik gegen die Monumente der populären Kultur zu richten.

Bereits der raffinierte erste Satz von Musils Essay - „Als Papa Tennis lernte, reichte das Kleid Mamas bis zu den Fußknöcheln“ (Musil 2000: 685) - verweist auf die Zurschaustellung des Körpers im Sport, denn er liefert nicht nur eine grobe Zeitangabe, die einen vergleichenden Blick auf die Gegenwart des Textes erlaubt, sondern bildet diese Zeit an der Rocklänge der Mutter ab. Die Perspektive des Textes ist damit vom ersten Moment an durch das Sehen - auf die damals freilich noch verhüll- 
ten Beine - bestimmt. Darüber hinaus erscheinen die Eltern als Paar in klassischen Geschlechterrollen, denn während „Рapa“ handelnd eingeführt wird, erscheint von „Mama“ nur ihr Kleid, das die Frau als Gesehene und gemessen an der Aktivität des Vaters als am Geschehen Unbeteiligte zeigt. Sie ist aber auf dem sich eröffnenden Schauplatz des Textes immerhin schon anwesend, sie ist möglicherweise die erste, die „Papa" beim Tennis zuschaut. Die Zeitform der Vergangenheit verdeutlicht, dass hier mehr in Bewegung geraten ist, als es der Satz im ersten Moment verrät. Die Leserinnen und Leser können vor ihrem geistigen Auge bereits den Fortgang der Zeit und die kürzer werdenden Röcke ergänzen, so dass sich der Übergang zur kulturellen Moderne als Sichtbarmachung des bewegten Körpers darstellt. Nicht zufällig nennt Musil in seinem Essay Sport und Mode in einem Atemzug. In seinem Artikel Frauenlob hat er beispielsweise „die Entkleidung der Frau durch den Sport“ (Musil 2000a: 804) als wichtigste Veränderung in den Geschlechterbeziehungen karikiert. Tatsächlich hingen die Veränderungen der Mode um die Jahrhundertwende, insbesondere die der Damenmode, mit den Anforderungen, die der Sport an die Bekleidung stellte, unmittelbar zusammen. So eroberten Frauen der höheren Schichten schon Ende des 19. Jahrhunderts das Fahrrad und den Tennisplatz. Die sportlichere Mode wurde damit generell zum Symbol größerer Bewegungsspielräume und Handlungsmöglichkeiten für Frauen (vgl. dazu den Beitrag von Burcu Dogramaci in diesem Band).

Auch bei Musil gerät die zu Beginn des Essays deutlich ausgestellte Geschlechterdifferenz mit dem Sport in Bewegung. „Mama“ trägt einen Glockenrock und eine Bluse mit einem ,hohen, engen Umlegekragen“ (Musil 2000: 685) als ,Zeichen einer Gesinnung, die bereits anfing, sich von den Fesseln zu befreien, die dem Weibe auferlegt sind“ (ebd.). Auch „Papa“ trägt diesen Kragen, „der ihn am Atmen hinderte“ (ebd.). Schwerfälligkeit und Aufbruchstimmung dieser Jahre zeigen sich noch bis zum Grund des Schuhwerks der Eltern: „An den Füßen schleppten beide nicht selten hohe braune Lederschuhe mit zolldicken Gummisohlen, und ob Mama außerdem noch ein Korsett zu tragen hätte, das bis an die Achselhöhlen reichte, oder sich mit einem kürzeren begnügen dürfte, war damals eine umstrittene Frage“ (ebd.: 685f.). Diese Frage ist als deutlicher Hinweis auf die Diskussionen um die Reformmode im Zuge der Lebensreformbewegung Ende des 19. Jahrhunderts zu verstehen, die sich weitgehend parallel zur Ausbreitung des Sports in Deutschland entwickelte (zur Lebensreform vgl. Andritzky/Rautenberg 1989 und Barlösius 1997; zur Reformmode vgl. Schultze-Naumburg 1910).

Als Reaktion auf die Lebensbedingungen in den Städten wurde hier der Weg zu einer möglichst naturgemäßen Lebensweise gesucht. Dabei 
bildete die ,Natur' des Körpers das Zentrum lebensreformerischer Überlegungen, die anders als im Sport als Gegenpol zum modernen Leben konzipiert wurde. Diese Differenz taucht auch in einer Tagebuchnotiz Musils auf, die den Wechsel der Moden thematisiert und aus der Erinnerung den hohen Umlegekragen als groteske Mischung aus dem steifen Uniform- und Halskragen der Biedermeierzeit und dem flachen Umlegekragen der Lebensreformer beschreibt. Dieser wurde, wie Musil abschätzig vermerkt, von „Turnern, Moralisten u.ä.“ getragen und hatte „den demonstrativen Charakter der natürlichen Lebensweise, Schlichtheit, Ablehnung des gesellschaftlich Vornehmen, u. nicht den des sportlichen Schicks“ (Musil 1983: 925f.). Dieser die Sportmode bejahende Eintrag macht die Stellung des frühen Sports zwischen bürgerlichliberalem Selbstbewusstsein, reformerischen Neuerungen und exklusivem Vergnügen sichtbar.

\section{II}

Während sich der Sport in England auf dem Land entwickelte und auch unter den Kritikern der Industrialisierung Verbreitung gefunden hatte, erwuchs seine Modernität in Deutschland aus seinem städtischen Charakter (vgl. Eisenberg 1999: 434). Es waren die sich am oberen und unteren Rand des Bürgertums ausdifferenzierenden Schichten der Großstädte, die zuerst Sport betrieben. Die Entwicklung einer großstädtischen Lebensweise mit ihren neuen Berufsfeldern und dem dazugehörigen Freizeitverhalten ebenso wie die Herausbildung einer neuen Wahrnehmung von Bewegung gingen mit der Etablierung der englischen sports Hand in Hand. In Deutschland repräsentierte der Sport die Hektik und ,Nervosität' modernen Lebens. Er brachte den modernen Zeitgeist zum Ausdruck, der auch die Mode kennzeichnete. In dieser Konstellation bedeutet die Anerkennung des Sports in Anschluss an Baudelaire eine Aufwertung des Flüchtigen und Zufälligen, die für die großstädtische Kultur kennzeichnend ist.

Dieses moderne Moment wurde durch das historische Zusammentreffen von Sportentstehung und Hochindustrialisierung verstärkt. Denn während sich die englische Sportbewegung parallel zur ersten industriellen Revolution formiert hatte, erfolgte dieser Schritt auf dem Kontinent zeitgleich mit ihrer zweiten Phase, der Rationalisierung und Verwissenschaftlichung der Produktion (vgl. ebd.: 435). Entsprechend sind in der Geschichte des deutschen Sports von Anfang an Sport und Technikbegeisterung eng verbunden. Diese Begeisterung sicherte etwa Auto- oder Fahrradrennen, aber auch Flugschauen stets großen Publikumszulauf. 
Gegenüber den Errungenschaften der modernen Welt verhält sich das Interesse am Sport affirmativ, da er ihre Prinzipien bejaht. Der Leistungs- und Wettkampfgedanke im modernen Sport ermöglichte die für viele befreiende Vorstellung, dass Leistung entgegen ständisch geprägten Einstellungen und dem System der Patronage auch zum dominierenden Faktor im öffentlichen Leben werden könnte. Dieser Tendenz der Moderne standen dagegen Teile der Lebensreform- und der Jugendbewegung eher ablehnend gegenüber.

Darüber hinaus ist die Herausbildung des Sports in eine Reihe mit solchen Erscheinungen zu stellen, die seit der Mitte des 19. Jahrhunderts zu einer auf Sichtbarkeit sich konzentrierenden, modernen Kultur beitragen. Dazu gehören der Zirkus, Völkerausstellungen, Freak-Shows sowie die Entwicklung des modernen Tanzes. Bei allen Unterschieden ist ihnen gemeinsam, dass es hier um die Inszenierung und Präsentation des bewegten menschlichen Körpers geht. Die genannten Präsentationsformen setzen die Überschreitung des Üblichen und nicht selten des Möglichen in Szene; sie thematisieren damit körperliche Normen und Grenzen, deren Überschreitung auch eine der Herausforderungen des Sports darstellt.

In Wien fanden diese Formen der Unterhaltung und Schaustellerei im 19. und frühen 20. Jahrhundert im Prater statt, insbesondere im sogenannten „Wurstlprater“, der seinen Namen der Figur des Hanswurst verdankt und damit auf komödiantische, körperbetonte Unterhaltungsformen zurückverweist. Im Prater traten unter anderen Artisten, behaarte Menschen, Dicke und Dünne, Riesen und Zwerge auf. Athleten, Ringkämpfer und ,starke Männer‘ zeigten sich seit Ende des 19. Jahrhunderts „regelmäßig im Prater bei Zirkusvorstellungen, im Unterhaltungsprogramm bei Festveranstaltungen und in eigens organisierten Ringkampfkonkurrenzen“ (Storch 1993: 18). Auch die ersten Wiener Kinos befanden sich im Prater (vgl. Horak/Maderthaner 1997: 102).

Wie viele Sportarten kam auch das Tennis im letzten Drittel des 19. Jahrhunderts von England auf den Kontinent. 1877 hatte erstmalig das Turnier von Wimbledon stattgefunden. ${ }^{3}$ Schon zwei Jahre später gründete sich in Bad Homburg der erste deutsche Tennisklub (vgl. Eisenberg 1999: 193). 1888 wurde in England die Lawn Tennis Association ins Leben gerufen, der bereits 1909 ,,hunderte englischer Tennisklubs und alle bedeutenderen Tennis-Vereinigungen des Festlandes angehören“ (Nordhausen 1909: 430). Interessanterweise wird in der zeitgenössischen Beurteilung für den enormen Erfolg des Tennis in Europa die Be-

3 Der Eintrag „Lawn-Tennis“ im Band Moderne Körperkultur spricht in diesem Zusammenhang bezeichnenderweise von ,in Szene gehen“ (vgl. Nordhausen 1909: 430). 


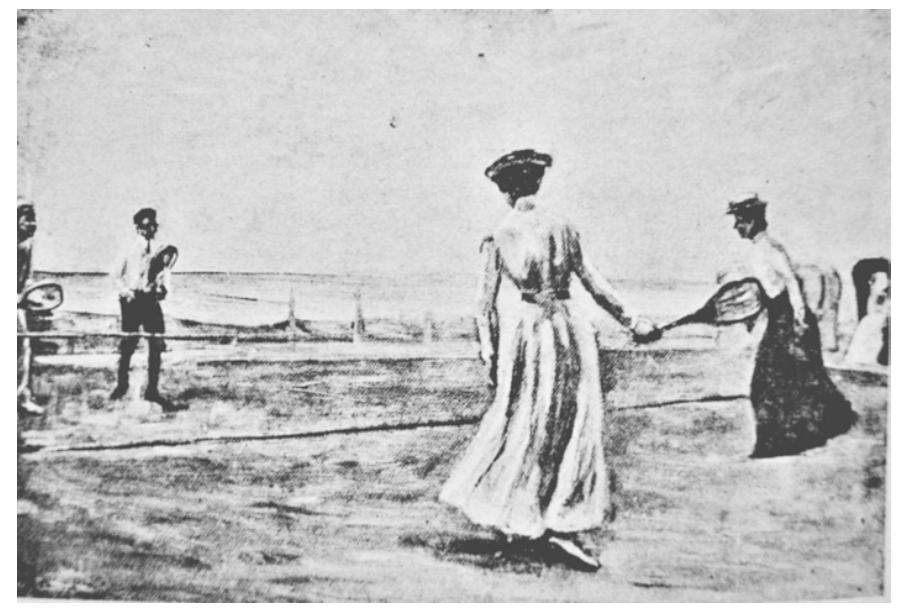

Abb. 2: Max Liebermann: Tennis.

teiligung des ,zarten Geschlecht[s]“ in Anschlag gebracht. Frauen wird die „Erlangung einer dem männlichen Partner im allgemeinen nicht nachstehenden, im Einzelfalle diesen nur zu oft überbietenden Spielgeschicklicheit" (ebd.: 431) attestiert. Und der Verfasser des hier zitierten Handbucheintrags von 1909 spricht gar von „Frauenbewegung im gegenständlichen Wortsinne“ (ebd.). ${ }^{4}$ Wird Tennis zunächst vor allem in der Sommerfrische gespielt, wird es als Teil einer zunächst vorwiegend großbürgerlichen Sportgeselligkeit bald auch in der Großstadt unentbehrlich.

Genau diese Entwicklung zeichnet Musils Text nach: Mit ihren schweren Schuhen spielten die Eltern noch auf ,romantischen Tenniswiesen“, zu denen Maulwurfshügel und Grasbüschel gehörten (Abb. 2). Mit dem Tennisboom wurde der moderne Hartplatz geschaffen, wodurch - so Musil - „ein ernster Zug in den Sport kam“ (Musil 2000: 686), der nicht nur dem beschleunigten Tempo der Ballwechsel geschuldet sein dürfte. So führte die Einrichtung moderner Tennisplätze zu einer Perfektionierung der Spieltechniken und der dazugehörigen Regeln: „Die Figuren verschwanden, die man anfangs hatte sehen können, wie sie, scharf visierend, mit turnerischer Geschicklichkeit das Racket einem Flugball entgegenstießen, und es bildeten sich überraschend schnell die Schläge aus, die heute noch gebraucht werden [...]. Auch die Listen des

4 Christiane Eisenberg hat herausgearbeitet, dass sich das Tennisspiel vor allem als gutbürgerlicher Heiratsmarkt empfahl (vgl. Eisenberg 1999: 199204). 
Spiels waren bald beisammen und fertig; nur nannte man sie damals noch nicht Taktik und Strategie" (ebd.). Aus individueller Geschicklichkeit wurden Schlagtechniken, aus spielerischen Tricks Strategien. Aus diesem sprachlich erzeugten Bedeutungszuwachs zieht der Autor den Schluss: „Seit Papa Tennis lernte, hat sich also immerhin einiges geändert, aber es betrifft mehr die Bewertung der Leibesübungen als diese selbst. [...] Was den Sport zum Sport gemacht hat, ist also nicht so sehr der Körper als der Geist" (ebd.: 687; Hervorhebung im Original). Musils Kritik richtet sich gegen die die Sportausübung zunehmend begleitenden Diskurse, die das Sportgeschehen mit Bedeutung überfrachten und die Wahrnehmung der Sportler bestimmen. Dabei gehen die Normierung der Körpertechniken des Sportlers und die Herausbildung öffentlicher Kennerschaft Hand in Hand, woraus eine klare Trennung von Sportausübenden und Sportpublikum folgt. Diese Trennung wird durch den Bau von großen Sportarenen - wie dem Stadion im Prater - verstärkt. So ist die Entstehung des Zuschauersports unlösbar mit der Entwicklung des professionellen Sports verbunden, der durch festgelegte Regeln, Zeitabläufe und Orte definiert ist, die im Wettkampf in der Arena seine spezifische ästhetische Qualität zur Geltung bringen.

\section{III}

Während Musil den organisierten Wettkampfsport kritisiert, attestiert er der gehobenen „Gesellschaft“, im Tennis „Spuren der ursprünglichen Moral" bewahrt zu haben. Diese Moral zeigt sich, wie schon der Beginn der Textes markiert hatte, an der Kleidung. „Wenn man von einer anderen Sportstätte auf einen Tennisgrund kam, so war das, sofern man einen empfänglichen Blick für Kleidung hatte, nicht anders, als ob man von einem offenen, hellen Platz in einen hochstämmigen Wald träte“ (Musil 2000: 686). Der Wald wirkt hier wie ein Vorhang, der die Blicke der Zuschauer verweigert und die Spieler selbst ihrem eigenen, exklusiven Vergnügen überlässt. Darüber hinaus steht der Wald aber auch für den Einklang von Spiel und Natur, der durch den Sport als Massenspektakel gestört wird. Denn die Professionalisierung des Sports drängt auf die öffentliche Zurschaustellung und Vermarktung der Körper in einer entsprechend gestalteten Umgebung, in der Regel also dem Stadion.

Die damit verbundene, fundamentale Veränderung kultureller Normen bringt Musil in einem bemerkenswerten Satz auf den Punkt: „Hier [im Tennis] reichten die Röcke noch bis zur halben Wade und die Taille bis zu den Handgelenken, als sich der Dreß anderswo längst schon auf die Größe eines Bogens Briefpapier, wenn nicht gar einer Eintrittskarte 
zusammengezogen hatte“ (ebd.). Mit der Entblößung des Körpers vollzieht sich endgültig der Wechsel von der bürgerlich geprägten, schriftzentrierten Hochkultur zu einer massenhaften Zuschauerkultur, die hier sinnfällig demonstriert wird: Wird mit dem Briefbogen das Symbol individueller Selbstverständigung und damit die Grundlage der bürgerlichen Literaturentwicklung - gleichsam ihr Gründungsdokument - noch einmal zitiert, so ist doch die Ablösung durch die Eintrittskarte unausweichlich, die keine noch so verschämte Lektüre übrig lässt und den nackten Körper zur Schau stellt, der auf das Spektakel verweist, ein Spektakel, das letztlich vor allem ökonomischen Gewinn bringen soll. Diese Nacktheit ist kein „Geheimnis des Garderobenschranks“ mehr, „das man nur selten tragen darf, weil es immer das gleiche bleibt“ (ebd.: 687), sondern Teil des neuen Sportgeists, der die Körperkultur als profitable Zuschauerkultur offensiv in Szene setzt und sich an der ,bloßen“ Zahl erfreut.

\section{IV}

Im Triumph des Sports erblickt Musil nicht nur einen Sieg über die Kunst, sondern auch einen Sieg über die Natur. Denn der Geist der Nacktheit entreißt nicht zuletzt dem Prater seine Geheimnisse. Der Sieg des Sports schlägt sich sowohl im Erscheinungsbild der Körper als auch im Wandel der Praterwiesen nieder. Mit dem Stadionbau thematisiert Musil in Als Papa Tennis lernte neben der Perfektionierung und Professionalisierung des Sports den Wandel im Verhältnis zur Natur, das der Körperschau vorausgeht. So war das Spiel der Eltern eingebettet in eigenes Naturerleben, wobei im Prater „Ideal und Wirklichkeit“ ausnahmsweise noch ,im Einklang“ waren: „Denn das war [...] ein stundenweiter Naturpark mit herrlichen alten Wiesen, Büschen und Bäumen; eine Landschaft, in der man sich als Mensch nur zu Gast fühlte; [...] aus irgendeinem Empfinden heraus, daß es doch noch etwas anderes als $\mathrm{Ku}$ gelstoßen oder Autofahren bedeute, wenn sich der Mensch langsam, ja sogar oftmals stehenbleibend oder sich setzend, in einer Umgebung bewegt, die ihm Empfindungen oder Gedanken eingibt, für die sich nicht leicht ein Ausdruck finden läßt“" (Musil 2000: 688). Während sich in diesem Empfinden Natur- und Selbsterfahrung harmonisch verbinden, erscheinen die Sportarenen als „Siegesplätze über die Natur“, die gegenüber der Umgebung verschlossen sind (vgl. Abb. 1). Nirgendwo zeige sich ,der lächerliche Anspruch der Leibesübungen, eine Erneuerung des Menschen zu sein, so naiv, so protzig, so instinktsicher“ (ebd.). 
Der von Musil kritisierte ,Geist des Sports‘ verkennt die vielfältigen, ,naturgegebenen“ Möglichkeiten sinnlichen Empfindens, da seine Wirklichkeit in festen Bahnen verläuft. Die Erneuerung des Menschen ist protzig und naiv zugleich, weil sie als Programm auftritt, wobei die äußere Natur lediglich zur Belebung der inneren Natur des Menschen dient (vgl. Gamper 2001: 52). Die ästhetische Erfahrung der Natur wird hier durch ein Verständnis von Natur abgelöst, die analog zum Sportkörper zunehmend entblößt, als nacktes Draußen erscheint. Das Stadion im Prater schließlich wird als moderne Zurichtung und Zivilisierung der Landschaft gedeutet, deren instrumentell verstandene ,Natur' sich in der Vorstellung eines technisch disziplinierten ,neuen` Menschen spiegelt.

Dieser Durchorganisierung des gesellschaftlichen Lebens, die Musil als kulturellen Paradigmenwechsel deutet, fällt subjektives Naturerleben ebenso zum Opfer wie künstlerische Qualität. Sarkastisch berichtet der Essay, „was weiter geschehen wird, wenn der letzte Baum des Wiener Praters Mitglied eines Sportvereins sein wird. Denn hier liegt bereits ein bemerkenswerter Vorschlag der Künstlerverbände vor, diese bloß vegetierenden Mitglieder zu Boden zu schlagen und, einstweilen wenigstens im Stadion, durch einen ,Denkmalshain“ zu ersetzen“ (Musil 2000: 690). Musil attackiert die angesichts der modernen Entwicklung in die Defensive geratenen Künstler für ihren Opportunismus gegenüber dem Sportbetrieb. Denn dergestalt gekappt und entwurzelt sind die Bäume nicht mehr Teil der Natur, sondern Objekt der Kunst, die sich dem Geist der Nacktheit unterwirft, in dem sie Tatsachen schafft. Darüber hinaus kritisiert Musil, dass die Kunst sich ihre Grundlage entzieht, die traditionell in der Naturnachahmung besteht, während sie den „Denkmalshain“ als „Wiedergeburt des Leibes“ anpreist (ebd.): „Die Not der bildenden Kunst ist groß, und das mag im Augenblick vieles rechtfertigen. Aber auch das Unvermögen, einen Akt zu bilden, den wir als unseren Ausdruck ansehen könnten, ist groß, und seit einem Menschenalter hat man darum die menschliche Plastik bald durch Walzen gezogen, bald unter Dampfhämmer gesetzt, aber ohne Erfolg, und wenn nun die Kunst, die uns einen Körper geben soll, nichts Schöneres und Tieferes findet als die Körper von athletischen Spezialisten oder überhaupt die von Athleten, so ist das zweifellos ein großer Triumph des Sports über den Geist" (ebd.).

Die damit noch einmal proklamierte Erneuerung des Menschen führt Musil schließlich auf die individuelle Sportausübung zurück, die gleichzeitig das Doppelgesicht des Sports deutlich macht: „Auf solche Ideen wäre ich bei meinen naiven körperlichen Anstrengungen seinerzeit gewiß niemals verfallen. Ich war fast ganz und gar ungeistig, nur um am nächsten Tag geistig frisch zu sein. Es kam mir beim Ringen wenig See- 
lisches in den Sinn, und wenn ich mich wie ein Tier betrug, so war mir eben gerade das erwünscht. Ich bin heute noch der Meinung, daß Geistesabwesenheit außerordentlich gesund ist, wenn man Geist besitzt, unter anderen Voraussetzungen jedoch auf die Dauer recht gefährlich!“ (ebd.: 690f.) Um geistig frisch zu sein, bedarf es nicht des organisierten Sportgeists. Der Gewinn des subjektiven Erlebens in der körperlichen Bewegung droht vielmehr durch zu hohe Sinnansprüche überfrachtet zu werden. Für das kollektive Erleben des Publikums wird dagegen gerade - so Musils Vorwurf - die vermeintliche Bedeutung des Sports als lukratives Spektakel in Szene gesetzt: „Aber wozu noch länger vom Geist des Sportsmanns reden, besteht doch das ganze Geheimnis darin, daß der Geist des Sports nicht aus der Ausübung, sondern aus dem Zusehen entstanden ist! Jahrelang haben sich in England Männer vor einem kleinen Kreis von Liebhabern mit der nackten Faust Knochen gebrochen, aber das war so lange kein Sport, bis der Boxhandschuh erfunden worden ist, der er es gestattete, dieses Schauspiel bis auf fünfzehn Runden zu verlängern und dadurch marktfähig zu gestalten“ (ebd.: 691; Hervorhebung im Original).

Erst das Publikum und festgelegte Regeln machen die Prügelei zum Sport, die gleichzeitig den Gesetzen eingängiger Inszenierung folgt. Das Sportspektakel wird zur Sensation, weil es sich nicht länger im kleinen Kreis - wie es auch der Geselligkeit beim Tennis entsprach -, sondern im Rahmen eines Massenpublikums vollzieht. Mit dieser Trennung von Sportausübung und Sportbetrachtung geht bei Musil allerdings ein auffälliger Wechsel der Sportarten einher, der auch auf soziale Vorbehalte gegenüber diesen Zuschauern schließen lässt. So diskutiert der Autor das Verhalten der Publikumsmasse an den Beispielen Boxen und Fußball: „Zweiundzwanzig Männer kämpfen mit der Mäßigung von Berufsmenschen um einen Fußball und einige Tausende, von denen die meisten einen solchen Ball niemals berührt haben, geraten in die Leidenschaft, die sich die Ausübenden ersparen. So entsteht der Geist des Sports. Er entsteht aus einer umfangreichen Sportjournalistik, aus Sportbehörden, Sportschulen, Sporthochschulen, Sportgelehrsamkeit, aus der Tatsache, daß es Sportminister gibt, daß Sportleute geadelt werden, daß sie die Ehrenlegion bekommen, daß sie immerzu in den Zeitungen genannt werden, und aus der Grundtatsache, daß alle am Sport Beteiligten, mit Ausnahme von ganz wenigen, für ihre Person keinen Sport ausüben, ja ihn möglicherweise sogar verabscheuen" (ebd.; Hervorhebung im Original).

Mit der Professionalisierung der Sportler korrespondiert die Herausbildung eines Publikums, das selbst nicht sportlich sein, wohl aber die Regeln des Sportgeschehens kennen muss. Auch hier zeigt sich noch einmal der historische Bogen, den Musil schlägt: Denn die ersten $\mathrm{Zu}$ - 
schauer im Sport waren die anderen Mitspieler oder Mitstreiterinnen. Mit der Etablierung der Regeln und der Institutionalisierung des Sports wandelt sich auch das Zuschauerverhalten bzw. das Publikum insgesamt. In diesem Entwicklungsprozess zeigt sich eine Unterscheidung, die beispielsweise im Theater als etablierter Präsentationsform längst selbstverständlich ist - nirgendwo wird von einem Theaterbesucher etwa erwartet, dass er selbst musiziert oder schauspielert (es sei denn, dies ist Teil der Inszenierung). Die Herausbildung der Differenz zwischen Ausübenden und Zuschauern ist hinsichtlich des Zusammenspiels von Sport und Künsten zentral, denn hier liegt nicht nur ein wichtiger Ansatzpunkt dafür, Sport und Kunst überhaupt miteinander zu vergleichen, sondern auch die zentrale Voraussetzung für eine ästhetische Bestimmung des Sports, wie sie Hans-Ulrich Gumbrecht und Martin Seel in Grundzügen entwickelt haben. ${ }^{5}$

Damit hat der Sport von Anfang an ein Publikum, wie es sich avantgardistische Theatermacher gewünscht haben, und er hat dazu die nötigen Räumlichkeiten, nämlich Arenen und Stadien, die das Spielfeld umgeben und damit die Bewegung der Körper in den Mittelpunkt der Betrachtung rücken. Nicht umsonst haben Theaterkünstler zu Beginn des 20. Jahrhunderts immer wieder auf Sportveranstaltungen zurückgegriffen, um ihre Vorstellungen und Erwartungen an Theaterbauten, Inszenierungen und das Publikum zu präzisieren. So strebte beispielsweise Max Reinhardt eine Arenabühne als ,Volkstheater' an, für die der Berliner Zirkus Schumann umgebaut wurde. Walter Gropius entwarf für Erwin Piscator ein „Totaltheater“, das in sich vollkommen variabel war, da sich Bühne und Zuschauerraum ineinander verschieben ließen (vgl. Fischer-Lichte 1997: 16). Bertolt Brecht forderte 1926 schlicht Mehr guten Sport im Theater: „Unsere Hoffnung gründet sich auf das Sportpublikum. [...] Die Verderbtheit unseres Theaterpublikums rührt daher, daß weder Theater noch Publikum eine Vorstellung davon haben, was hier vor sich gehen soll. In den Sportpalästen wissen die Leute, wenn sie ihre Billette einkaufen, genau, was sich begeben wird: und genau das begibt sich dann, [...] nämlich, daß trainierte Leute mit feinstem Verantwortungsgefühl, aber doch so, daß man glauben muß, sie machten es hauptsächlich zu ihrem eigenen Spaß, in der ihnen angenehmsten Weise ihre besonderen Kräfte entfalten“ (Brecht 1992: 119f.).

5 Kennzeichnend für eine solche Ästhetik des Sports ist die Produktion von Präsenz, die u.a. durch die Interaktion von Sportlern und Publikum zustande kommt (vgl. dazu Gumbrecht 1998 und Seel 1996; vgl. zur Bedeutung von Präsenz Gumbrecht 2004). 
In formaler Hinsicht zeigt Musils mehrfacher Rückbezug auf den Prater das essayistische Umkreisen von Gedanken an, die sich in der Tat „nicht leicht" ausdrücken lassen und dem forschen Siegeszug des Sportsgeists ein retardierendes Moment einschreiben. Dieses literarische Verfahren lässt sich selbst als Form der Kontingenzerzeugung verstehen, das Inhalte des Diskurses mit den von diesem ausgeschlossenen Elementen konfrontiert (vgl. Gamper 2001: 36) und damit das Sportgeschehen aus verschiedenen Perspektiven, auch auf Umwege abschweifend ins Visier nimmt. So heißt es beispielsweise: „Ehe ich aber von diesem berühmten Geist beginne, muß ich eine Geschichte erzählen, die weitab davon anfängt, jedoch bald dahinführt“ (Musil 2000: 687). Damit stellt Musil seine These auch in der Strategie des Essays aus: Den Geschichten, die die Literatur - und sei es in der Erinnerung - bereithält, stehen die nackten Tatsachen hinter den „Eintrittsschranken“ der Stadien bereits gegenüber. In diesem weitläufigen Gedankenraum sind unbeobachtete Begegnungen möglich, und der Einlass ist frei.

Dem literarischen Raum korrespondiert der Gang durch die unbelassene Natur des Praters. Musils essayistische Haltung stellt den herrschenden Sportdiskurs in Frage, der analog zur Lebensreform in der sportlichen, körperlichen Bewegung eine Annäherung an die Natur feiert (vgl. auch Gamper 1999: 145). Denn die proklamierte Bewegung in der frischen Luft ist bereits durch bloße Naturbeherrschung bestimmt, während die Bewegung, die Musils Essay nachzeichnet, vom Prater als Ort einer intensiven, aber unbestimmten Naturerfahrung ausgeht. Dadurch sieht der Text ein friedliches Nebeneinander verschiedener Erfahrungsmöglichkeiten am Werke, das erst die sportliche Organisation der Masse im Stadion aufhebt: „Jahrhundertelang haben sich Leute als Schnell- und Dauerläufer, Springer und Reiter sehen lassen, aber sie sind ,Gaukler" geblieben, weil ihre Zuschauerschaft nicht sportlich ,durchorganisiert" gewesen ist" (Musil 2000: 691). Im Nebeneinander dieser Erscheinungen bleibt Raum für die Kontingenz des Ereignisses, für das Vergnügen gegenüber dem organisierten Spektakel.

Hierin liegt schließlich auch Musils eigenes Interesse am Sport begründet, der ,das Wesen des Ich [...] in den Erlebnissen des Sports aus dem Dunkel des Körpers“ (ebd.: 690) hervorleuchten sieht. „Wie weitläufig wäre allein schon (obwohl sie gegeben werden kann) die Erklärung des Wunders, daß man auf die Entfernung des Anlaufs vorausbestimmen kann, mit welchem Fuß man abspringen wird!“ (ebd.) In diesem „Wundergefühl“ (ebd.: 689) erkennt Musil eine Möglichkeit augenblickshaft aufscheinender Einigkeit des eigenen Ich, die sich ent- 
sprechend nur unbewusst einstellt. Dazu schreibt er in Durch die Brille des Sports: „Das ist einer der größten Reize des Sports. Im Augenblick der Ausführung springen $\mathrm{u}[\mathrm{nd}]$ fechten dann die Muskeln $\mathrm{u}[\mathrm{nd}]$ Nerven mit dem Ich, nicht dieses mit ihnen, u[nd] sowie nur ein etwas größerer Lichtstrahl von Überlegung in dieses Dunkel gerät, fällt man schon aus dem Rennen. Das ist aber nichts anderes als ein Durchbruch durch die bewußte Person, eine Entrückung“ (Musil 2000b: 793).

Als eigentlich geistige Anregung des Sports bezeichnet Musil in Als Papa Tennis lernte - im Gegensatz zu der von ihm scharf kritisierten Durchorganisierung, in der er sowohl eine Instrumentalisierung der Körper als auch eine der Natur sieht, die beide dem „Wunder“ entgegenstehen - die „Bekanntschaft mit den Fehlleistungen, welche der wahrnehmbaren Müdigkeit voranschleichen; man lernt das eigentümliche Schweben zwischen zuviel und zuwenig Fleiß kennen, die beide schädlich sind, den gewöhnlich ungünstigen Einfluß der Affekte auf die Leistung und andererseits die beinahe mirakulöse Natur des besonders guten Gelingens, wo der Erfolg sozusagen schon vor der Anstrengung da ist“" (Musil 2000: 689f.).

Die Faszination des Sports liegt also gegenüber der institutionalisierten Inszenierung des Körperschauspiels im Ereignis des gelingenden, nicht genau voraussehbaren Augenblicks. Damit beschreibt Musil eine Möglichkeit des Sports, die er allerdings schon im Prozess der Entstehung des modernen Wettkampfsports wieder schwinden sieht. „Man fühlt ein Vakuum, in das sich der Sport stürzt. Man weiß eigentlich nicht recht, was sich da stürzt, aber alle reden davon, und so wird es wohl etwas sein: so ist immer das an die Macht gekommen, was man ein hohes Gut nennt" (ebd.: 691). Musils Kritik an der pompösen Diskursivierung des Sports, die auf das Programm eines neuen Menschen und die Organisation von Publikumsmassen zielt, kann auch als kritische Beschreibung einer kommerzialisierten Zuschauerkultur gedeutet werden. Diese Kritik schließt die Faszination des Autors durch die subjektive Bewegungserfahrung und den ästhetischen Reiz des Sportgeschehens keineswegs aus, im Gegenteil: Musil hat in der skizzierten geistigen Durchdringung des sinnlichen Erlebens ein bedeutendes Potential für die Herausbildung moderner Individualität erkannt. In seiner pessimistischen Einschätzung des modernen Sportbetriebs hat er sich gleichwohl als hellsichtig erwiesen. 


\section{Literaturverzeichnis}

Andritzky, Michael/Rautenberg, Thomas (Hg.) (1989): „Wir sind nackt und nennen uns du“. Von Lichtfreunden und Sonnenkämpfern. Eine Geschichte der Freikörperkultur, Gießen: Anabas.

Barlösius, Eva (1997): Naturgemäße Lebensführung. Zur Geschichte der Lebensreform um die Jahrhundertwende, Frankfurt a.M., New York: Campus.

Brecht, Bertolt (1992): Mehr guten Sport. In: Ders.: Werke. Große, kommentierte Berliner und Frankfurter Ausgabe, hg. v. Werner Hecht, Jan Knopf, Werner Mittenzwei, Klaus-Detlef Müller, Bd. 21, bearb. v. Werner Hecht u. Marianne Conrad, Berlin, Weimar: Aufbau, Frankfurt a.M.: Suhrkamp, S. 119-122.

Eisenberg, Christiane (1999): „English sports“ und deutsche Bürger. Eine Gesellschaftsgeschichte 1800-1939, Paderborn, München, Wien: Schöningh.

Fischer-Lichte, Erika (1997): Die Entdeckung des Zuschauers: Paradigmenwechsel auf dem Theater des 20. Jahrhunderts, Tübingen, Basel: Francke.

Fleig, Anne (2004): „Die Geburt des Sports aus dem Geist des Zuschauens. Robert Musils Essay ,Als Papa Tennis lernte““. In: Dieter Mersch (Hg.), Sport - Inszenierung - Ereignis - Kunst, Kiel: Forum der Muthesius-Hochschule, S. 40-48.

Gamper, Michael (1999): „Im Kampf um die Gunst der Masse“. In: Hans-Georg Arburg/Michael Gamper/Dominik Müller (Hg.), Popularität. Zum Problem von Esoterik und Exoterik in Literatur und Philosophie, Würzburg: Königshausen \& Neumann, S. 135-163.

Gamper, Michael (2001): „Ist der neue Mensch ein ,Sportsmann“? Literarische Kritik am Sportdiskurs der Weimarer Republik“. Jahrbuch zur Kultur und Literatur der Weimarer Republik 6, S. 35-71.

Gumbrecht, Hans-Ulrich (1998): „Die Schönheit des Mannschaftssports: American Football im Stadion und im Fernsehen“. In: Gianni Vattimo/Wolfgang Welsch (Hg.), Medien - Welten - Wirklichkeiten, München: Fink, S. 201-228.

Gumbrecht, Hans-Ulrich (2004): Diesseits der Hermeneutik. Die Produktion von Präsenz, Frankfurt a.M.: Suhrkamp.

Horak, Roman/Maderthaner, Wolfgang (1997): Mehr als ein Spiel. Fußball und populare Kulturen im Wien der Moderne, Wien: Löcker.

Musil, Robert (1983): Tagebücher, hg. v. Adolf Frisé, Reinbek: Rowohlt.

Musil, Robert (2000): Als Papa Tennis lernte. In: Gesammelte Werke II, hg. v. Adolf Frisé, erw. Neuausgabe, Reinbek: Rowohlt, S. 685-691. 
Musil, Robert (2000a): F.F.Bl. Frauenlob. In: Gesammelte Werke II, hg. v. Adolf Frisé, erw. Neuausgabe, Reinbek: Rowohlt, S.804f.

Musil, Robert (2000b): Durch die Brille des Sports. In: Gesammelte Werke II, hg. v. Adolf Frisé, erw. Neuausgabe, Reinbek: Rowohlt, S. 792-795.

Nordhausen, Richard (1909): Moderne Körperkultur. Ein Kompendium der gesamten modernen Körperkultur durch Leibesübungen, Leipzig: Verlag von J. J. Arnd.

Schultze-Naumburg, Paul (1910): Die Kultur des weiblichen Körpers als Grundlage der Frauenkleidung, Jena: Eugen Diederichs.

Seel, Martin (1996): Ethisch-Ästhetische Studien, Frankfurt a.M.: Suhrkamp.

Storch, Ursula (1993): Das Pratermuseum. 62 Stichwörter zur Geschichte des Praters, Wien: Museen der Stadt Wien. 